\title{
Semantic Reconciliation in Interoperability Management through Model-Driven Approach
}

\author{
Frédérick Bénaben ${ }^{1}$, Nicolas Boissel-Dallier ${ }^{1,2}$, Jean-Pierre Lorré $^{2}$, and Hervé Pingaud ${ }^{1}$ \\ ${ }^{1}$ Mines Albi - Université de Toulouse, France \\ ${ }^{2}$ Petals Link - EBM WebSourcing \\ \{frederick.benaben, nicolas.boissel-dallier\}@mines-albi.fr, \\ herve.pingaudamines-albi.fr \\ \{nicolas.boissel-dallier, jean-pierre.lorre\} @petalslink.com
}

\begin{abstract}
The MISE Project (Mediation Information System Engineering) aims at providing virtual enterprises with a Mediation Information System (MIS) in charge of supporting interoperability in a collaborative network. MISE proposes an overall MIS design method according to a model-driven approach, based on model transformations. This MIS is in charge of (i) information management, (ii) services sharing and (iii) behavior orchestration. Semantic issues are accompanying those objectives: How to deal with information reconciliation? How to ensure the matching between business activities and technical services? How to identify workflow among business process? This article aims first, at defining these semantic gaps along the whole MISE approach. The second part of this article presents how some specific solutions have been found in the specific context of crisis management (French funded project ISyCri). Finally, the current and future works concerning semantic reconciliation (in a generic non « 1 to $1 »$ context) will be introduced.
\end{abstract}

Keywords: interoperability, semantic reconciliation, model-driven engineering, information system.

\section{Introduction}

Collaborative networks support large diversity of collaboration forms, including virtual enterprises [1]. Virtual enterprises and the associated collaborative network are strongly dependent from their ability to manage collaboration and to assume the involved interoperability functions: information exchange, activities coordination and processes orchestration. Furthermore, Information Systems (IS) and computed systems are assuming both the roles of interface of organizations (external and internal exchanges) and functional engine of organizations (driving processes and business activities). Therefore, the previously listed interoperability functions must be supported by these IS. A collaborative network must consequently be able to provide one homogeneous IS, dealing with all the potentially heterogeneous IS of its components (partners of the collaborative network).

The MISE project (Mediation Information System Engineering), presented in [2] and [3], aims at defining a model-driven design approach of a MIS (Mediation 
Information System), in charge of interoperability functions. This MIS could be considered as a third-part based on Service-Oriented Architecture (SOA) principles, allowing existing IS to work fluently altogether, according to a common behavior, without any special effort. This goal is fully compliant with the definition of interoperability given in [4] and [5]. The MIS design framework defined in MISE, dealing with business requirements and logical considerations in order to deploy a technical mediation solution should necessarily succeed in the business-to-technical alignment: the abstract solution (proposed at the business and/or logical level) must be precisely implemented by the concrete solution (deployed at the technological level). The semantic attributes must be appropriately defined at the abstract level and rigorously taken into account at the concrete level.

Following the previous considerations, the main objective of this article is to present the semantic issues embedded into the MISE project, according to two horizontal levels: abstract (business/logical) and concrete (technology) among three vertical layers: informational (information vs. data), functional (activity vs. service) and behavioral (process vs. workflow).

The second section of this article introduces the MISE project and the associated general principles. The third section identifies semantics issues. The fourth section presents specific solutions that have been used in the French funded project based on MISE results (ISyCri - Interoperability of Systems in Crisis situations). The fifth section outlines perspectives and current work to deal with these semantic issues.

\section{Overview of MISE Project}

The following global presentation of the MISE project will be based on three main parts: (i) overall big picture of the design approach, (ii) model transformation principles and (iii) presentation of each step of the design approach.

\subsection{Overall Big Picture of the Design Approach}

This design approach might be seen as a dive into abstraction layers. Consequently it is based on model-driven engineering and on the associated model transformation concepts [6]. The general principle is therefore structured into three steps:

1. Gathering a structured knowledge on the considered collaboration (partners, roles, goals, services, data, etc.) in order to instantiate a specific ontology. By applying deduction rules on this knowledge, a collaborative process model is deduced (as described in [7]). This is the CIM level (Computation Independent Model), corresponding to the business layer.

2. The knowledge embedded in this collaborative process is then used through model transformation mechanisms in order to propose a MIS structure (as described in [8]) at the PIM level (Platform Independent Model), corresponding to the logical layer.

3. Finally, a technical model of the MIS is build, based on the knowledge embedded in the PIM and on the technological target platform, which is an ESB (Enterprise Service Bus) at the PSM level (Platform Specific Model), corresponding to the technological layer. 
The two first steps concern the abstract level while the third step is dedicated to the concrete level (which is a useful point considering the semantic issues to come). The following picture illustrates this global MDE design approach:

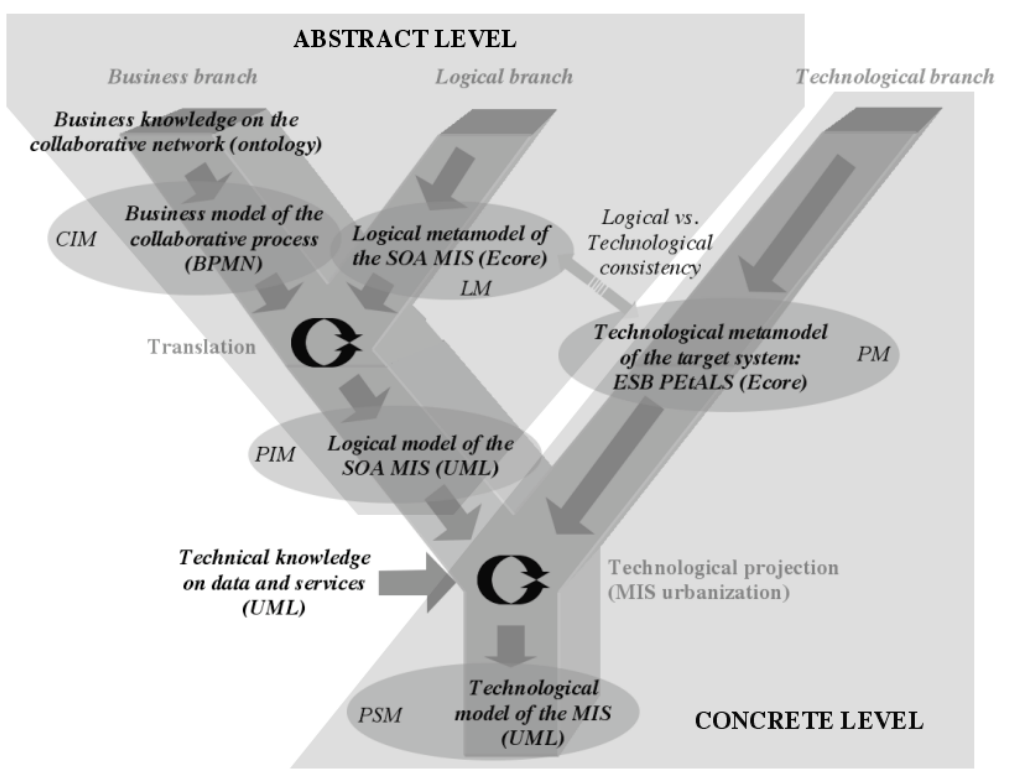

Fig. 1. "Big Picture" of the MISE design approach

Before detailing this MDE approach, we will focus on the required theoretical elements concerning model transformation principles.

\subsection{Model Transformation Principle}

Considering our model-driven issue, the first crucial point of the presented approach is model transformation. This issue concerns each of the three previously presented steps (business / logical / technical). A source model is used (built according to a source metamodel (MM)) to obtain a target model (respecting a target MM). The key point is that the source MM shares part of its concepts with the target MM. Note that the two spaces, source and target, have to be partially overlapping in order to allow model-morphism. As a consequence, the source model embeds a shared part and a specific part. The shared part provides the extracted knowledge, which may be used for the model transformation, while the specific part should be saved as capitalized knowledge in order not to be lost. Then, mapping rules (built according to the overlapping conceptual area of MMs) can be applied onto the extracted knowledge in order to provide the transformed knowledge. That transformed knowledge and an additional knowledge (to fill the lack of knowledge concerning the non-shared part of concepts into the target MM) may be finally used to create the shared part and the specific part of the target model. The general theoretical framework of all these model transformations may be synthesized as follow: 


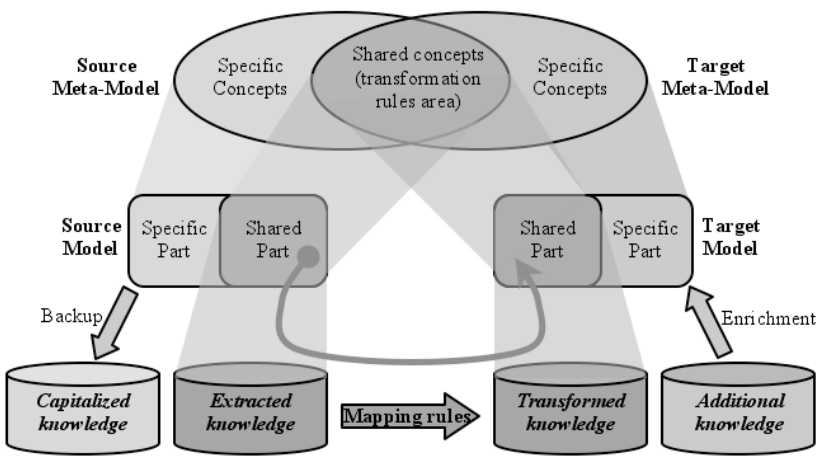

Fig. 2. Model transformation principle

Note that both, capitalized knowledge and additional knowledge may be empty depending on the considered model transformation case.

\subsection{Presentation of Each Step of the Design Approach}

On a semantic point of view, the MISE design approach includes two levels (abstract and concrete). On a model transformation point of view, it includes three steps (business, logical and technical). This section presents these steps in order to introduce the semantic gaps to solve in the MISE approach (see [3] for more details).

- Business step (Abstract level): The objective is to transform a characterization of the collaborative situation into the business model of a collaborative process. According to section 2.2, the source model is a network characterization, based on a network ontology (respecting a network MM which is the source MM). The additional knowledge is a repository of business activities, containing all the available functions of partners. As detailed in [9], this repository may be the Process Hand Book (see [10]). The target MM is the collaborative process MM described in [7], while the mapping rules may therefore be the ones fully described in [8]. There is no specific capitalized knowledge in this model transformation step. The obtained target model is finally a collaborative process model, dedicated to support dynamically the described collaborative situation.

- Logical step (Abstract level): The objective is to transform a business model of a collaborative process into the model of the logical structure of the MIS. According to section 2.2, the source model is the previously obtained collaborative process model, based on the collaborative process MM (which is the source $M M$ ). There is no specific additional knowledge, which shows that this model transformation is still at the abstract level. The target MM (SOA UML $\mathrm{MM}$ ) and the mapping rules are fully described in [7]. There is no specific capitalized knowledge in this model transformation step. The obtained target model is finally a SOA logical UML model, dedicated to present the logical architecture of the MIS in charge of supporting the collaborative situation. 
- Technical step (Concrete level): The objective is to transform the model of the logical structure of the MIS into the technical model of the MIS (usable for an ESB deployment). According to section 2.2, the source model is the previously obtained SOA logical UML model, based on the SOA architecture MM (which is the source MM). The additional knowledge contains all the technical elements concerning services and data (knowledge embedded into WSDL files). The target $M M$ is the UML technical architecture of the MIS. The mapping rules are described in [3]. There is no specific capitalized knowledge in this model transformation. The obtained target model is a UML model, describing the technical structure of the MIS (based on the deployment of an ESB).

The following section will focus on the semantic issues of the MIS design. In such a design schema, strongly based on model-transformation principles, the semantic gaps are clearly linked to the additional knowledge. Actually, this knowledge brings new elements that have to be identified and correlated to the existing knowledge and the target MM in order to be appropriately integrated in the target model.

\section{Semantic Issues in MISE Project}

According to the previous section, the main semantics gaps might be, first at the business model design step and second at the transition between logical and technical MIS models (two steps where additional knowledge is included). The first semantic issue will not be considered in this article, as it is more a matter of transition between problem description and potential solution. The second semantic issue is more interesting due to the fact that it is a transition between abstract level and concrete level, aiming at finding technical elements to implement strictly logical components. Therefore, the global semantic framework of the MISE project is the following:

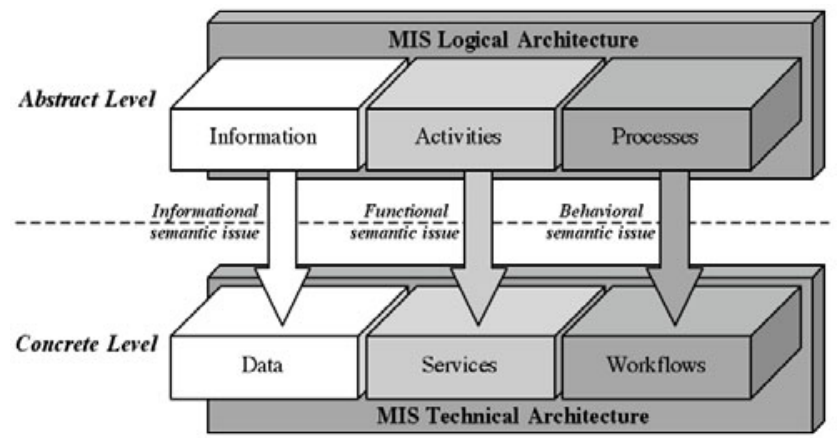

Fig. 3. Semantic framework of the MISE project

There are clearly three semantic issues, which, once solved, may allow to connect relevantly abstract level to concrete level: informational issue, functional issue and behavioral issue. These issues may be formulated as follows: How to deal with information reconciliation? How to ensure the matching between business activities 
and technical services? How to obtain workflow from business process? The first two questions deals with many-to-many issues. The objective is to map a set of business activities (respectively information) with a set of technical services (respectively data) during the model transformation (in order to define a technical way to implement precisely a theoretical solution). Furthermore, information are linked to activities (activities deal with information as inputs or outputs for example) and data are linked to services (similarly, services deal with data). The third question is more a problem of translation due to the fact that process defines the way activities and information should behave while workflow does the same with services and data.

\section{Specific Semantic Treatment in ISyCri Project}

The ISyCri project is a French funded project (ANR/06/CSOSG) dealing with Interoperability of Information Systems in Crisis situations. It is mainly focus on providing a crisis management cell with a MIS dedicated to ensure the collaboration between partners of the crisis cell (while these partners are assuming the collaboration with their people on site through their personal and specific channels). To reach that goal, the previous semantic issues had to be solved, in the crisis management context.

We will first explain the functional issue as far as it may be considered as the corepart, essential to understand both the informational issue and the behavioral issue. The solution adopted to deal with that key semantic issue is quite rough but well adapted to the considered field: The added knowledge of the business step is based on a repository of technical services. This is a strong short-cut to avoid semantic problems at the functional levels but it is quite realistic as far as, for partners of a crisis management cell, technical service are actually factual representation of business activities. For example, if policemen are able to establish on the crisis site a security perimeter (business activity), and if the MIS, orchestrating the collaborative workflow inside the crisis management cell, aims at starting that action, then, the MIS should call a service of the policemen IS (technical service) which will inform the policemen delegate inside the crisis management cell that it is time to establish the security perimeter. This technical service will therefore be a kind of interface, requesting the business activity of establishing a security perimeter. Schematically, in this case, instead of trying to match business activities with technical services, the chosen way oblige to select business activities among technical services. The matching is not necessary anymore. This direct way to deal with the functional semantic issue is mainly compliant with the specific context of crisis management (due to the previous consideration on proximity of technical services with business activities) but would not be adapted to other domains where the semantic distance between technical services and business activities would be more consequent.

As for the informational issue, first a part of the required matching is done through the previous mechanism as far as selecting business activities among a repository of technical services implies also to select inputs and outputs of this business activities. Furthermore, another principle is also in charge of that informational semantic reconciliation: Mediation services (inside the MIS) are in charge of translation and matching between data. If one output information of one business activity is conceptually an input of another business activity, it is necessary that the output of the 
corresponding technical service is correctly interpreted and used as the input of the following corresponding technical service. Therefore, some static matching tables have been built, according to the specific considered field. Mediation services have also been built in order to be able to read these tables and to use them in order to transform outputs of technical services into required inputs of other technical services:

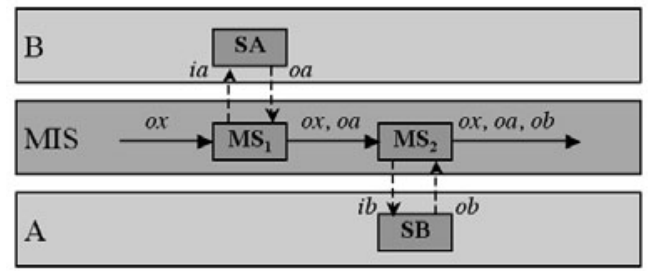

Where mediation services $M S_{1}$ and $\mathrm{MS}_{2}$ know, thanks to matching tables, that:

$o x=\left(o x_{1}, o x_{2}, o x_{3}\right)$

$i a=o x_{1}+o x_{3} \quad$ (built by $\left.M S_{t}\right)$ $o a=\left(o a_{1}, o a_{2}\right) \quad$ (result from $\left.S A\right)$ $i b=o x_{2}-o a_{1} \quad$ (built by $M S_{2}$ ) and are able to provide services with the required elements

Fig. 4. Mediation services principle

The informational semantic issue is then managed by the ability of mediation services to use pre-established semantic matching tables. Similarly to the functional solution, this is a result specifically dedicated to crisis management context. As a critical point, the design of matching tables should be automatically managed by knowledge management tools. Currently it is a manual work, thanks to the fact that, in crisis management situation, the existing emergency plans define formally the information required for each activity and where they can be found.

Finally, concerning the behavioral issue, there is no real semantic reconciliation due to the fact that the workflow design is based on a BPEL transformation tool, which proposes a BPEL file, orchestrating technical services and data (already semantically consolidated by the management of informational and functional issue). The behavioral issue is managed through the syntactic mapping (BPEL generation) and the preceding semantic reconciliations (informational and functional).

\section{Semantic Issues in MISE: Perspectives and Current Work}

Semantic issues have been identified and defined in MIS design context. Furthermore, some specific ways to solve these issues have been presented in the particular field of crisis management. In this case, we consider that semantic distance between business activities and technical service is small enough to select activities directly into a technical service repository. We also created static matching tables for the mediation service. This approach is only available with a few static services. These solutions are not satisfying for a general context. That is why we are currently working on semantic reconciliation in MISE.

In one hand, we aim to match business activities and technical services, considering granularity differences between abstract and concrete levels. It involves an "n-to-m" matching during the logical to technical transformation and ontology matching of concepts from different levels. In the other hand, we aim to enable on the fly data adaptation with automatic add of transformation services when necessary, in order to avoid manual matching. Service and data reconciliations require three phases: 
(i) knowledge modeling of technical and collaboration domain concepts, (ii) incorporating semantics into business and technical models thanks to existing or new standards, (iii) using semantic information to match services or messages. It is a major challenge in MIS design to find semantic correspondences between business activities and real web services as well as to configure messages transformations on the fly using semantic matchmakers.

In order to generalize its use to any collaboration network, our work focuses on existing standards such as SAWSDL, OWL-S, WSMO or WSMO-Lite which support efficiently development of Semantic Web Services [11]. Some of these standards allow semantic description of internal service behavior (e.g. internal process of orchestration service). On the other hand, by describing semantically business activities (through business ontologies), we plan to infer abstract profile of technical service (or set of services) able to assume these business roles. The final semantic reconciliation between services and abstract profile of inferred (set of) service(s) will bridge the gap between abstract and technical layers, through an "n-to-m" matching.

\section{References}

1. Camarinha-Matos, L.M., Picard, W.: Foreword. In: 5th IFIP International Conference on Virtual Enterprises (Pro-VE), vol. 283, pp. xv-xvi. Springer, Poznan (2008)

2. Benaben, F., Pingaud, H.: The MISE project: a first experience in Mediation Information System Engineering. In: 5th Italian Chapter of AIS (ITAIS), Paris (2008)

3. Benaben, F., Wenxin, M., Truptil, S., Lorre, J.-P., Pingaud, H.: Information Systems design for emerging ecosystems. In: 4th IEEE International Conference on Digital Ecosystems and Technologies, Dubai (2010)

4. Konstantas, D., Bourrieres, J.-P., Leonard, M., Boudjlida, N.: Preface. In: 1st International Conference on Interoperability of Enterprise Software and Applications (InterOp-ESA), pp. v-vi. Springer, Genova (2005)

5. Pingaud, H.: Prospective de recherches en interoperabilite: vers un art de la mediation. In: Plenary Lecture, 8th International Congress on Industrial Engineering (CIGI), Tarbes (2009)

6. OMG, MDA Guide Version 1.0.1, omg/2003-06-01 (2003)

7. Touzi, J., Bénaben, F., Pingaud, H., Lorré, J.-P.: A Model-Driven approach for Collaborative Service-Oriented Architecture design. International Journal of Production Economics 121(1), 5-20 (2009)

8. Rajsiri, V., Lorré, J.-P., Bénaben, F., Pingaud, H.: Knowledge-based system for collaborative process specification. Computers in Industry 61(2), 161-175 (2009)

9. Rajsiri, V., Lorré, J.-P., Bénaben, F., Pingaud, H.: Collaborative process definition using an ontolohy-based approach. In: 5th IFIP International Conference on Virtual Enterprises (Pro-VE), vol. 283, pp. 205-212. Springer, Poznan (2008)

10. Malone, T.W., Crowston, K., Herman, G.A.: Organizing business knowledge. In: The MIT Process Handbook, chs. 1, 3 (2003) ISBN 0-262-13429-2

11. Lausen, L., Lara, R., Polleres, A., de Bruijn, J., Roman, D.: Description: Semantic Annotation for Web Services, Semantic Web Services: Concepts, Technologies and Applications, pp. 179-209. Springer, Heidelberg (2008) 